\title{
E-9. Effects of Laser Radiation upon the Transplanted Brain Tumors
}

\author{
Kiyoo Kamikawa, Toru Hayakawa, Takuya Ikeda, \\ Ryotaro Kuroda, Yukitaka Ushro and Hiromasa Murui \\ Department of Neurosurgery Osaka University Medical School,
}

Since laser is monochromatic light, absorption of laser is expected to be increased by physiologic pigments and dyes in the irradiated tissues. Various dyes have been used as exogenous photosensitizer in laser research. Dyes increase the biomedical effects of laser energy. It is well-known that the absorption of dye injected intravenously takes place in tumor tissue of brain, while the blood-brain barrier limits the entrane of dye from blood to brain and consequently the normal brain tissue remains unstained. Those evidences indicate that there is a possibility of selective destruction of brain tumor with less damages of normal brain tissues by laser radiation. Experiments were designed to apply the laser for treatment of brain tumor. Acridine orange was used as a photosensitizer to argon gas laser because of the match in absorption of light.

$\mathrm{VX}_{2}$ tumor was transplanted in the cortex of rabbits and 4 days after the transplantation, $2 \mathrm{ml}$ of $1 \%$ acridine orange solution was given intravenously. On the next day additional $2 \mathrm{~m} l$ of solution was given several hours prior to radiation. Laser was $200 \mathrm{~mW}$ exit energy at $4880 \AA$ of wave length. Laser irradiated on the dural surface in the tumor-implanted area through a burr hole for $10 \mathrm{sec}$ to $15 \mathrm{~min}$. Animals were sacrificed one week after the radiation. Histological examination revealed that welldeliniated necrotic lesion had been made in the irradiated tumor tissue. The lesion made by radiation for $25 \mathrm{sec}$ was $2.2 \mathrm{~mm}$ in width and $1.9 \mathrm{~mm}$ in depth, while radiation for $10 \mathrm{sec}$ produced small lesion. However prolonged radiation for 15 min could not increase the bulk of lesion so much.

Less damaged lesions were found in tumor tissues irradiated by laser without dye. Photosensitizing effects of dye was proved in brain tumor by laser radiation, although laser with exit energy of 10 joule $/ \mathrm{cm}^{2} / \mathrm{sec}$ has thermal effect on irradiated tissues. One of the characteristic histological findings was pleomorphic changes of tumor cells in surrounding area of necrotic lesion. It must be clarified in further investigation that those pleomorphic changes indicate either malignant or degenerative. 